\title{
correspondence
}

\section{The toxicity of plutonium}

SIR,-We wish to comment on three recent publications, all originating in the United Kingdom, which have discussed the radiotoxicity of inhaled, insoluble plutonium compounds. In a recent article in Nature, Thorne and Vennart ${ }^{\prime}$ concluded that the previously accepted value for the maximum permissible annual intake (MPAI) of such compounds may be too high by a factor of about five. A similar conclusion had previously been reached in a Medical Research Council report ${ }^{2}$, and in a report ${ }^{3}$ by the Royal Commission on Environmental Pollution. Our main purpose here is to point out:

that the proposed changes in the MPAI and in the closely-related maximum permissible air concentration (mpc) do not necessarily imply any change in the present procedures for assessing doses and risks in situations where persons have known amounts of insoluble plutonium in their lungs;

- that although the article in Nature and the MRC report come to broadly the same final conclusions, the reasons for the conclusions are significantly different; and that the uncertainties in the calculations, to which attention is drawn in both papers, are as large as the proposed reduction factor;

- that the observed behaviour of the technologically-important high fired plutonium oxide in people who have accidentally inhaled such material is significantly different from the behaviour predicted from the lung model assumed in recent MPAI calculations.

Maximum permissible annual intake. Thorne and Vennart stress that the MPAI which they derive is intended for planning purposes ${ }^{1}$. We believe that most facilities in which plutonium oxide is handled are planned on the basis that there will be virtually no chronic exposure, and that this is also true in operational practice; hence the precise value of the MPAI (or the mpc) is somewhat irrelevant. However, exposures do occur from time to time, due to unplanned but identified incidents which usually cause the exposure of one or two individuals to quite high concentrations for a short time. In such situations, we believe that it is good practice to make the best estimates of intake, dose and risk to those specific individuals on the basis of measurements taken on them, with no reference to the concepts of MPAI (or $\mathrm{mpc}$ ). In fact, such a procedure is explicitly recommended ${ }^{4}$ in ICRP Publication 19 on the page following the one on which they describe the model used in Nature and the MRC report.

Uncertainties in the MPAI. Although the Nature article and the MRC report both conclude that a reduction in the MPAI of the order of a factor of five is to be recommended, the two sets of calculations show significant differences. For example, the respective estimates of risk of bone cancer differ by more than a factor of five and the risks of lung cancer by more than a factor of three. On reading the respective texts it is obvious where the differences lie (in the relative importance of the respiratory lymph nodes, in the quality factor for alpha particles and in the calculations of dose to the bone). Both publications acknowledge that the estimates of 'nominal risk' are open to criticism and that the data on which they are based can be interpreted in several ways. It is an unfortunate fact that such qualifications are often disregarded by those who draw conclusions from the final tabulated presentation.

The maximum permissible lung burden (MPLB). In the summary of conclusions (paragraph 526.2) of its sixth report, the Royal Commission on Environmental Pollution ${ }^{3}$ claimed that a reduction by a factor of five in the MPLB is already practised. This statement appears to have resulted from a misunderstanding; we believe that their statement was intended to apply to the MPAI. The conclusion stated in paragraph 526.2 does not follow from the section of the report in which the matter is discussed (i.e. paragraph 77). Neither the MRC report nor the Nature article explicitly suggested a reduction in the MPLB, and in our view, no such reduction is required in the case of high fired plutonium oxide.

ICRP lung model. As explained above, all of the recent calculations have been based on the use of the lung model of ICRP Publication 19. The parameters given in the model were derived mainly from animal data, and presumably represent at best the mean or median values of a number of widely differing experimental values. Consequently, as explained in the associated text of the ICRP publication, the model can only be used for deriving general exposure limits; to evaluate a specific exposure situation one should choose the data most relevant to that particular situa- tion. Measurements made on a substantial number of individuals $\mathrm{s}^{5,6}$, who had inhaled high fired plutonium oxide, show that the material initially deposited in the lung is removed much more rapidly than the ICRP model would suggest for what it calls a Class Y material. Moreover, experience here at Winfrith (to be published) has shown that the amount of material transferred from the lung to other parts of the body is also significantly less than the model would suggest; perhaps this is a consequence of the more rapid removal to which we have referred. As a consequence of these two factors, if risk estimates are made for those people who have inhaled high fired plutonium oxide, they are found to be a factor of about 5 lower than the risk estimates based on the use of the lung model, i.e. they are no higher than was previously supposed.

Discussion. We feel that the methods which have been used to derive new values for the MPAI are unduly pessimistic for high-fired plutonium oxide. That fact in itself does not worry us unduly. What does worry us is that people may be tempted to conclude, by the use of the same lung model, that in every case in which a person inhales insoluble plutonium, the risks to bone and liver are comparable to those of lung; and hence to conclude that the MPLB should be reduced. We believe that in the case of high fired plutonium oxide, the previously established idea that the lung is the critical organ remains substantially correct.

D. RAMSDEN

T. F. JOHNS

Atomic Energy Establishment

Winfrith, Dorset, UK

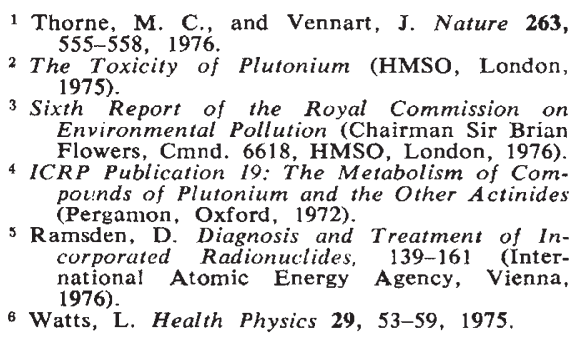

\section{Registering Russian mail}

SIR-Apropos of your article "Keeping in touch with Soviet colleagues" (10 February, page 484), it is my experience that correspondence with them is expedited by using registered mail.

Culham Laboratory, UK 